\title{
Clinical Interface of Macular Retinal and cpRNFL Thickness, OND by OCT and Visual Field for Glaucoma Diagnosis Macula Research for Glaucoma Diagnosis
}

\author{
Dovile Buteikiene $^{1}$, Asta Kybartaite Ziliene ${ }^{* 2}$, Justina Budeniene ${ }^{1}$ and Ingrida Januleviciene ${ }^{1}$ \\ ${ }^{1}$ Department of Ophthalmology, Medical Academy, Lithuanian University of Health Sciences, UK \\ ${ }^{2}$ Laboratory of Biophysics and Bioinformatics, Neuroscience Institute, Lithuanian University of Health Sciences, UK
}

Received: May 26, 2018; Published: June 07, 2018

*Corresponding author: Asta Kybartaite Ziliene, Laboratory of Biophysics and Bioinformatics, Neuroscience Institute, Medical Academy, Lithuanian University of Health Sciences, UK

Abstract

Background: Assessment of clinical interface of macular retinal thickness and circumpapillary retinal nerve fiber layer (cpRNFL) thickness, optic nerve disc (OND) parameters using optical coherence tomography (OCT)and visual field mean deviation in the large scale case-control study were combined to produce a better diagnostic strategy for early glaucoma detection, monitoring and prognosis.

Materials and Methods: It was the case-control study comprising 45-74 years old men and women of 499 healthy and198 patients with primary open angle glaucoma (POAG). All the study subjects underwent full ophthalmological investigation and OCT scanning of the macula, cPRNFL and OND. The statistical analysis was done.

Results: The macular retinal and cpRNFL thickness in sectors, except for the fovea, showed statistically significant thinning in the POAG group compared with the control group. The sectoral macular retinal thickness averages had stronger correlation with respective sectoral cpRNFL thickness averages in the case of POAG. It is possible to make the prognosis that odds ratio to have POAG increases by 3.5 times when retinal thickness average of inferior outer macula is less than $219 \mu$. The odds ratio of having cup/disc vertical ratio bigger than 0.577 significantly increased by 2.8 times if inferior outer macular retinal thickness was less than $219 \mu$. The odds ratio of having visual field deeper than MD -3.55dB significantly increased 3.5 times if inferior inner + outer macular retinal thickness was less than $237 \mu$.

Conclusion: Thinning of the macular retinal thickness in the sectors had significant clinical interface with changes in cpRNFL, visual field loss and OND cupping in glaucoma and may represent a surrogate indicator of retinal ganglion cell loss. Macular thickness measurements with OCT may provide a new approach for the detection and monitoring of glaucomatous damage.

Keywords: Macular Retinal Thickness; Circumpapillary Retinal Nerve Fiber Layer Thickness; Optic Nerve Disc; Primary Open Angle Glaucoma; Optical Coherence Tomography

Abbreviations: cpRNFL: Circumpapillary Retinal Nerve Fiber Layer; OND: Optic Nerve Disc; OCT: Optical Coherence Tomography; POAG: Primary Open Angle Glaucoma; RGC: Retinal Ganglion Cells; RNFL: Retinal Nerve Fiber Layer; VF: Visual Field; IPL: Inner Plexiform Layer; IOP: Intraocular Pressure; UCVA: Uncorrected Visual Acuity: BCVA: Best Corrected Visual Acuity; LogMAR: Logarithm of the Minimum Angle of Resolution; VRI: Vitreoretinal Interface; RPE: Retinal Pigment Epithelium; TI: Temporal Inner Macula; SI: Superior Inner Macula; NI: Nasal Inner Macula; II: Inferior Inner Macula; TO: Temporal Outer Macula; SO: Superior Outer Macula; NO: Nasal Outer Macula; IO: Inferior Outer Macula; SPSS: Statistical Package for Social Science; ROC: Receiver Operating Characteristic

\section{Background}

Glaucoma is an optic neuropathy that is characterized by progressive loss of the retinal ganglion cells (RGC) and their axons in the retinal nerve fiber layer (RNFL), thinning of the neuroretinal rim in the optic nerve disc (OND) and visual field (VF) defects [1]. The circumpapillary retinal nerve fiber layer (cpRNFL) and OND

assessments are the mainstay of glaucomatous structural measurements. The macula has been suggested as an alternative scanning location for glaucoma diagnosis.

Analysis of cpRNFL and OND measurement using optical coherence tomography (OCT) was generally superior to macular assess- 
ment. However, macular measurement showed better glaucoma diagnostic performance and progression detection capability in some specific cases, for example in the cases of high degree of myopia, OND abnormalities (druze, pit, papilledema, paripapillary changes etc.), which suggests that these measurements may be combined to produce a better diagnostic strategy. Also clinical decision-making is complicated due to a large variation in OND size and shape within a population and also among populations [1-3], various combinations of OND glaucomatous damage and the inter- and intra-operator variability was reported in many other studies [4].

Compared to the OND, the macula is a relatively simple structure that is devoid of large vessels. The macula offers several potential physiological and anatomical advantages for glaucoma evaluation. First, the RNFL is composed of the RGC axons, and therefore assessment of the RGC may be a more direct method for measuring glaucomatous damage than cpRNFL thickness [1]. Furthermore, more than $50 \%$ of the RGC of the entire retina are located within 4-5mm of the macula center [5-7] and cell bodies are stacked up to six layers thick [6-10].Thus scanning the macula allows sampling of the majority of the RGC [1] and small losses of RGC bodies (which along with the RNFL constitute more than $30 \%$ of the retinal thickness) are detectable by analyzing total retinal thickness [8]. Total macular thickness is a surrogate measure of tissue thickness loss due to glaucoma in the absence of other macular pathology (which might affect other layers of the retina) [8]. Changes in total macular thickness loss in glaucoma reflect the loss of the RNFL, RGC and the inner plexiform layer (IPL) tissues that are lost in glaucoma [8]. The macula shape, more specifically the RGC layer, is generally less variable among healthy individuals than other diagnostically important structures, such as the RNFL and OND [1].

The total macular thickness (internal limiting membrane to retinal pigment epithelium) is easily and accurately measured by optical reflective devices such as the OCT due to the high level of reflectivity from these two boundary regions of the retina [8]. The Stratus OCT measured the central $6 \mathrm{~mm}$ perifoveal area by acquiring data from six radial line scans intersecting at the fovea and created a map of macular thickness which interpolated the data between the lines [8]. The aim of this study was to assess the clinical interface of macular retinal thickness and cpRNFL thickness, OND parameters using OCT and visual field mean deviation for glaucoma diagnosis in the large scale analytical observation case-control study.

\section{Materials and Methods \\ Participants of the Study}

The study was approved by the Kaunas Regional Human Research Ethics Committee, Lithuania and all participants signed an informed consent form in accordance with the tenets of Declaration of Helsinki. Detailed study and its methods were described previously [11]. In brief, it was the analytical observation case-control study. The control group comprised 45-74 years old men and women. Inclusion criteria in our study for the control group were as follows:

a) No congenital or acquired optic nerve pathology, absence of macular pathology which might affect layers of the retina; b) A participant was not previously diagnosed with glaucoma;

c) There were no defects deeper than the $\mathrm{p}<2 \%$ in the visual field (tested by Frequency Doubling Technology Screening);

d) Signal strength of OCT images of the macula, cpRNFL and OND was greater than 5 .

The case group comprised 45-74 years old men and women with primary open angle glaucoma (POAG). Inclusion criteria for the case group were as follows:

a) Intraocular pressure (IOP) was higher than $21 \mathrm{~mm} \mathrm{Hg}$ by Schotz'o tonometer (using the weights $5.5 \mathrm{~g}$ ) at the time of POAG diagnosis;

b) The OND glaucomatous structural changes were described in the outpatient's card;

c) Glaucomatous visual field defects were approved by two Humphrey SITA Standard (24-2) tests;

d) The signal strength of OCT images of the macula, cpRNFL and OND was greater than 5 .

\section{Ophthalmologic Investigation}

At the beginning, the following data of the questionnaire were filled in: age, sex, medical history of the eye pathology and comorbidity. Uncorrected visual acuity (UCVA) and the best corrected visual acuity (BCVA) were tested at 4 meters using a Logarithm of the minimum angle of resolution (LogMAR) chart [12]. The refraction was performed by means of computerized refractometer ACCUREF-K9001 (Shin-Nippon, Japan). The IOP was measured with a Schiotz tonometer (Riester, Germany) under local anesthesia with $0.5 \%$ proxymetacaini. Biomicroscopy of the anterior and posterior eye segments was performed by a standard slit lamp. Lens opacity grading was performed using the Lens Opacities Classification System III (LOCS III) according to a written standard protocol [13]. The control group participants underwent frequency doubling visual field screenings (N-30-5 Frequency Technology Screening, Humphrey Matrix, Carl Zeiss Meditec). The case group participants underwent 2 standard automated perimeter threshold visual field tests (Central 24-2 Threshold Test stimuli III, White, SITA - Fast / Standard, Humphrey Field Analyzer II, Carl Zeiss Meditec).

\section{Optical Coherence Tomography}

OCT of the macula, cpRNFL and OND was performed with the Stratus OCT 3000 (software version 4.0, Carl Zeiss Meditec) [14]. It is a computer-assisted optical instrument that generates cross sectional two dimensional (2D) images (tomograms) of the layers of the retina with axial and transverse resolutions of $10 \mu \mathrm{m}$ and $20 \mu \mathrm{m}$, respectively $[1,14]$. The Stratus OCT calculates retinal thickness as the distance between the vitreoretinal interface (VRI) and the junction between the inner and outer segment of the photoreceptors, which is just above the retinal pigment epithelium (RPE) [14]. It calculates RNFL thickness as the distance between the VRI and the RNFL posterior boundary. The retinal and RNFL thickness algorithms use an iterative process, applying various techniques in 
a fixed and logical progression, first to obtain rough estimates of the layer boundaries, and then to refine the estimates. The algorithm calculations operate on reflectivity values (in decibels) in the individual A-scans. These algorithms take advantage of the Stratus OCT high resolution, resulting in a highly refined objective measurement of retinal or RNFL thickness [14]. The Stratus OCT evaluates the signal strength of frozen or saved scan images and assigns a number from 0 (worst) to 10 (best), which it displays in the signal strength field [14].

\section{Macula}

The "Fast Macular Thickness Map" protocol was performed for macular thickness acquisition [14]. This scan protocol assesses the macular thickness on the basis of six $6 \mathrm{~mm}$ long radial scans at equally spaced angular orientations (each scan rotated by $30^{\circ}$ ), with intersection in the foveolar region. 128 equally spaced samples (A-scans) are taken along each radial scan (fixed at $768 \mathrm{~A}$-scan totals). At each of these 128 sample points it makes 1024 equally speed measurements over a depth of $2 \mathrm{~mm}$ (786432 integrated data points at all) [14].

The "Retinal Thickness Tabular" was selected as the analysis protocol, and the calculation of macular thickness map was based on the 6mm "Fast Macular Thickness Map" scanning protocol [14]. The map was composed of 9 sectors in 3 concentric circles, each with a diameter of $1 \mathrm{~mm}$ (central), $3 \mathrm{~mm}$ (inner), and $6 \mathrm{~mm}$ (outer). The inner and outer rings were divided into four quadrants as follows: Temporal inner macula (TI), Superior inner macula (SI), Nasal inner macula (NI), Inferior inner macula (II), Temporal outer macula (TO), Superior outer macula (SO), Nasal outer macula (NO), Inferior outer macula (IO) (Figure 1).

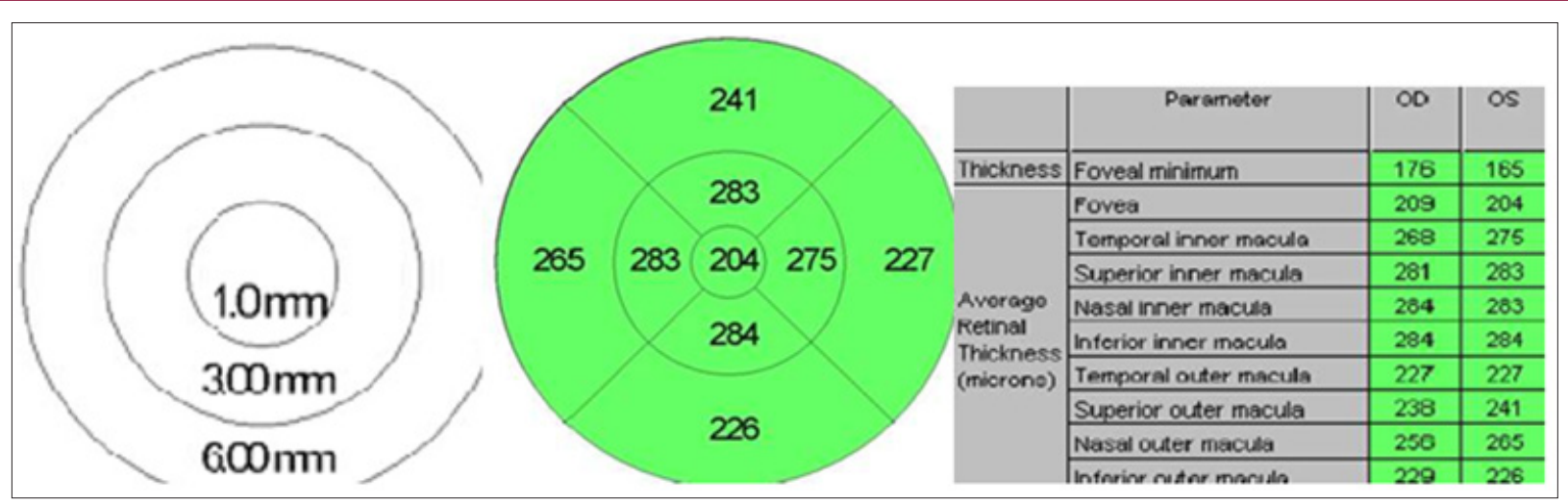

Figure 1: Macular thickness map of the left eye(OS).Optical coherence tomographic imaging was performed using a 6 radial scans (6mm diameter) centered on the fovea to generate a macular thickness map.

\section{Circumpapillary Retinal Nerve Fiber Layers}

The cpRNFL was measured using the "RNFL Thickness (3.4)" protocol, which perform 3 circle scans of diameter $3.4 \mathrm{~mm}$ around the OND with $512 \mathrm{~A}$-scan points for each circle scan. The 3 RNFL measurements were then averaged. Measurements were then assessed using "RNFL Thickness Average" analysis protocol. The output includes data of the RNFL thickness at the Nasal, Superior, Temporal and Inferior quadrants of the peripapillary region of the OND (Figure 2).

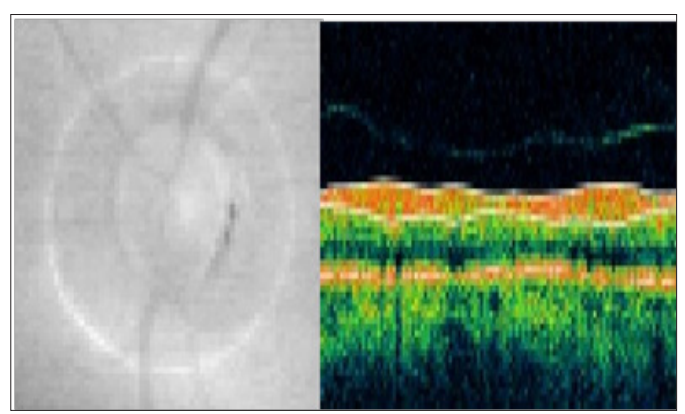

Figure 2: CircumpapillaryRNFL thickness of the left eye (OS).Optical coherence tomographic imaging was performed using $3,4 \mathrm{~mm}$ diameter peripapillary measurement circle.

\section{Optic Nerve Disc}

OND scans were acquired by the "Fast Optic Disc" protocol. This protocol consists of a series of six equally spaced $4 \mathrm{~mm}$ radial line scans through a common central axis. With each scan pass, the Stratus OCT captures $128 \mathrm{~A}$-scans (768 A-scans total). Each A-scan consists of 1024 data points over $2 \mathrm{~mm}$ of depth. Thus, the Stratus OCT integrates 786432 data points to construct a cross-sectional 2D image of OND anatomy. OND analysis was carried out by "Optic Nerve Head" protocol. The analysis automatically calcu- lates quantitative OND parameters (optic nerve disc, cup, rim areas and cup/ disc ratios) for each of the six radial line scans and the output enables to interactively assess and measure them using each scan individually and a composite of all scans.

For each scan in the group, the "Optic Nerve Head" analysis detects the anterior surface of the RNFL and the RPE. It detects the RNFL surface by searching each A-scan from anterior to posterior until it finds reflectivity above a threshold value. From below the RNFL surface, it searches each A-scan posteriorly for the highest rate of change in reflectivity to find the RPE surface. Having determined these boundaries, the algorithm detects and measures all features of disc anatomy based on the anatomical markers (disc reference points) on each side of the disc where the RPE ends. It 
locates and measures the Disc Diameter by tracing a straight line between the two disc reference points and measures Cup Diameter on a line parallel to the disc line and offset anteriorly by 150 micrometers. It determines Rim Area using the cup line as a posterior boundary; for the rim lateral boundaries it uses lines extended from the disc reference points perpendicular to the disc line and up to the anterior surface of the disc. The results of these detection and measurement algorithms are displayed graphically on the scan image. In the output display, it is possible to adjust the placement of the disc reference points, and thus the resulting measurements. Optic Nerve Head analysis combines the analysis and measurement of each individual scan into a composite image and measurements of the whole OND. The outputs are displayed as OND individual scans and the results of composite image measurements (Figure 3). Acquired scans of the macula, cpRNFL and OND with signal strength of more than 5 were analized.

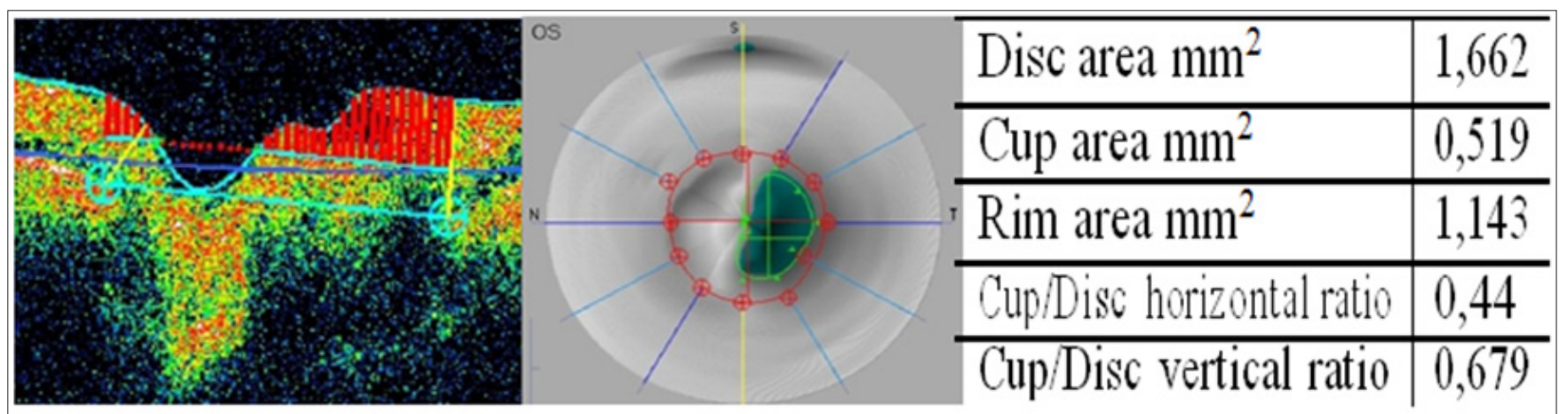

Figure 3: Optic nerve disc of the left eye (OS) parameterization by optical coherence tomography.

\section{Statistical Analysis}

The Statistical Package for Social Science (SPSS 22.0 Windows and Statistica 6.0) was used for statistical analysis. The quantitative variables were described as the arithmetic mean and standard deviation of $\mathrm{M}(\mathrm{SD})$. Continuous variable normality assumption was verified using the Kolmo- gorov-Smirnov test. When distribution was normal, the mean differences between independent samples of the two groups were assessed using the Student $(\mathrm{t})$ test. The means of more than two groups were compared using the dispersion analysis (ANOVA), for multiple pair-wise comparison Bonferroni test (post hoc) was applied. When the test of normality of the investigated variables was denied or variables were nonparametrical, the Mann-Whitney and Kruskal-Wallis test were used. The differences in the distribution of qualitative variables were assessed by chisquare test. The linear dependence of the variables was evaluated using the correlation coefficient. According to the distribution of variables, we used Pearson or Spearman correlation coefficient.

Receiver Operating Characteristic (ROC) curve analysis method was used for determination of optimal values of macula thickness parameters. The optimal values separated out the different groups Table 1: Characteristics of the study population. with the highest accuracy (minimal false negative and false positive). The binary logistic regression analysis was performed to determine odds ratio of participants with POAG. A two- sided p value less than 0.05 was considered to be statistically significant.

\section{Result}

A total of 697 subjects were enrolled in the study. The control group was formed by healthy participants $-71.6 \%$ of all subjects. The case group included POAG patients - $28.4 \%$ of all subjects (Table 1). There were 1339 eyes under research. An average quality of macula OCT test was 8.05(1.5), cpRNFL-9.18(1.1). Statistically significantly better quality of macula and cpRNFL OCT was in the control group than in the POAG group, respectively $\mathrm{p}=0.008$ and $\mathrm{p}<0.001$. There was no significant difference of the average retinal thicknesses at the fovea between the study groups. The macular retinal thickness in sectors (Temporal inner macula, Superior inner macula, Nasal inner macula, Inferior inner macula, temporal outer macula, Superior outer macula, Nasal outer macula, Inferior outer macula) and the total macular thickness (volume) showed statistically significant thinning in the POAG groups compared with the control group (Table 2).

\begin{tabular}{|c|c|c|c|}
\hline \multirow{2}{*}{ Parameters M(SD) } & \multicolumn{2}{|c|}{ The Study Groups } & \multirow[t]{2}{*}{ p-value } \\
\hline & Control $n=499 ;(n *=958)$ & POAG n=198; $\left(n^{*}=381\right)$ & \\
\hline Age, years & $65.3(8.5)$ & $65.1(9.0)$ & 0.8 \\
\hline Male/Female, in \% & $38.3 / 61.7$ & $26.8 / 73.2$ & $0.004^{*}$ \\
\hline BCVA by LogMAR & $0.15(0.21)$ & $0.19(0.21)$ & 0.004 \\
\hline Spherical equivalent of refraction, diopter & $1.03(2.24)$ & $0.52(2.29)$ & 0.001 \\
\hline Intraocular pressure, $\mathrm{mmHg}$ & $15.94(2.45)$ & $15.44(2.96)$ & 0.001 \\
\hline Visual field mean deviation, in decibel & & $-3.62(2.8)$ & \\
\hline Lens nuclear opacification (LOCS III ) & $1.5(0.63)$ & $1.63(0.47)$ & 0.001 \\
\hline Lens cortical opacification (LOCS III) & $0.78(0.99)$ & $0.88(0.98)$ & 0.02 \\
\hline Lens posterior subcapsularopacification (LOCS III) & $0.18(0.42)$ & $0.2(0.54)$ & 0.6 \\
\hline
\end{tabular}


M(SD): Mean Value and a Standard Deviation; BCVA by LogMAR: The Best Corrected Visual acuity by Logarithm of the Minimum Angle of Resolution; LOCS III: Lens Opacities Classification System III; POAG: Primary Open Angle Glaucoma; n: The Number of Subjects; $n^{*}$ : Number of Eyes; p*: p: Value in Groups

Table 2: The macular, cpRNFL thickness and OND parameters by OCT parameterization of the study groups.

\begin{tabular}{|c|c|c|c|c|}
\hline \multirow{2}{*}{$\begin{array}{l}\text { The Retinal Thickness of the Sectors } \\
\text { of the Macula in Microns, M(SD) }\end{array}$} & \multicolumn{3}{|c|}{ Study Groups } & \multirow[b]{2}{*}{$\mathbf{p}$} \\
\hline & Control $(n=945)$ & POAG $(n=380)$ & $\begin{array}{c}\text { Mean Difference [95\% CI of the } \\
\text { Difference] }\end{array}$ & \\
\hline Fovea & $205.82(32,53)$ & $204,87(38,89)$ & $0.95[-3.15-5.06]$ & 0.6 \\
\hline Temporal inner macula & $257.55(23.36)$ & $249.68(25.32)$ & $7.87[5.02-10.73]$ & $<0.001$ \\
\hline Superior inner macula & $268.26(22.45)$ & $260.78(25.43)$ & $7.47[4.69-10.26]$ & $<0.001$ \\
\hline Nasal inner macula & $267.43(23.86)$ & $261.9(24.68)$ & $5.53[2.66-8.41]$ & $<0.001$ \\
\hline Inferior inner macula & $267.38(22.42)$ & $258.21(28.24)$ & $9.18[6.29-12.07]$ & $<0.001$ \\
\hline Temporal outer macula & $217.68(18.66)$ & $208.32(19.03)$ & $9.36[7.12-11.6]$ & $<0.001$ \\
\hline Superior outer macula & $232.89(18.68)$ & $224.2(21.61)$ & $8.66[6.32-10.99]$ & $<0.001$ \\
\hline Nasal outer macula & $249.14(19.62)$ & 239.91(18.99) & $9.23[6.91-11.54]$ & $<0.001$ \\
\hline Inferior outer macula & $227.21(20.43)$ & $215.73(22.69)$ & $11.48[8.85-14.11]$ & $<0.001$ \\
\hline Total macula thickness & $243.7(18.92)$ & $235.96(21.54)$ & $7.75[1.26-5.26]$ & $<0.001$ \\
\hline Total macular volume $\left(\mathrm{mm}^{3}\right)$ & $6.75(0.49)$ & $6.5(0.54)$ & $0.25[0.19-0.31]$ & $<0.001$ \\
\hline $\begin{array}{l}\text { cpRNFL thickness at the sectors, in } \\
\text { microns, } M(S D)\end{array}$ & \multicolumn{2}{|c|}{ Control $(n=958)$} & POAG $(\mathrm{n}=381)$ & $\mathrm{p}$ \\
\hline Superior (S) & \multicolumn{2}{|c|}{$116.07(18.5)$} & $98.29(23.87)$ & $<0.001$ \\
\hline Nasal (N) & \multicolumn{2}{|c|}{$77.26(16.4)$} & $67.45(17.83)$ & $<0.001$ \\
\hline Inferior (I) & \multicolumn{2}{|c|}{$121.99(18.62)$} & $103.86(25.18)$ & $<0.001$ \\
\hline Temporal (T) & \multicolumn{2}{|c|}{$68.14(13.93)$} & 61.15(13.67) & $<0.001$ \\
\hline The average thickness (Avg.) & \multicolumn{2}{|c|}{$95.89(12.11)$} & $82.69(13.38)$ & $<0.001$ \\
\hline Optic nerve discparameters & \multicolumn{2}{|c|}{ Control (n=958) } & POAG (n=381) & $\mathrm{p}$ \\
\hline Disc Area mm² & \multicolumn{2}{|c|}{$2.14(0.41)$} & $2.2(0.45)$ & 0.04 \\
\hline CupArea mm² & \multicolumn{2}{|c|}{$0.55(0.41)$} & $0.86(0.58)$ & $<0.001$ \\
\hline Rim Area mm² & \multicolumn{2}{|c|}{$1.6(0.38)$} & $1.33(0.41)$ & $<0.001$ \\
\hline Cup/ Disc Horizontal Ratio & \multicolumn{2}{|c|}{$0.46(0.22)$} & $0.58(0.23)$ & $<0.001$ \\
\hline Cup/ Disc Vertical Ratio & \multicolumn{2}{|c|}{$0.44(0.2)$} & $0.56(0.22)$ & $<0.001$ \\
\hline
\end{tabular}

M(SD): Mean Value and a Standard Deviation; POAG: Primary Open Angle Glaucoma; n: number of eyes; p: value between groups; cpRNFL: circumpapillary Retinal Nerve Fiber Layer

The mean of the cpRNFL thickness and the means of the sectors (Superior, Nasal, Inferior, Temporal), were significantly lower in the POAG group (Table 2). The mean of OND area was significantly larger in POAG group compared with the control group. The means of optic cup are cup/ disc area ratio and cup/ disc vertical and horizontal diameter ratios were significantly larger, but the mean of NRR area was significantly smaller in POAG group. The sectoral macular retinal thickness averages had a stronger correlation with respective sectoral cpRNFL thickness averages in the case of POAG. The aver-

age linear correlation linked Superior outer macula with cpRNFL Superior, Inferior outer macula with cpRNFL Inferior, Inferior inner+outer macula with RNFL Inferior, Total macular volume with RNFL Inferior, Total macular thickness/ volume with RNFL thickness average (Table 3).

Table 3: The correlations of macular retinal and circumpapillary retinal nerve fiber layer thickness, rim area, visual field of the study groups.

\begin{tabular}{|c|c|c|c|}
\hline \multirow{2}{*}{$\begin{array}{l}\text { The Correlationsbetween the Macular Retinal and cpRNFL } \\
\text { Thickness, Visual Field, OND }\end{array}$} & \multicolumn{2}{|c|}{ Study Groups } & \multirow{2}{*}{$\mathbf{p}^{*}$} \\
\hline & Control $(n=945)$ & POAG $(n=380)$ & \\
\hline Inner macula & \multicolumn{2}{|c|}{ the correlation coefficient, $\mathrm{p}<0.001$} & \\
\hline Temporal inner macula with RNFL Temporal & 0.295 & 0.405 & 0.04 \\
\hline Superior inner macula with RNFL Superior & 0.188 & 0.365 & 0.002 \\
\hline Nasal inner macula with RNFL Nasal & 0.2 & 0.214 & 0.81 \\
\hline Inferior inner macula with RNFL Inferior & 0.273 & 0.407 & 0.013 \\
\hline
\end{tabular}




\begin{tabular}{|c|c|c|c|}
\hline Outer macula & \multicolumn{2}{|c|}{ the correlation coefficient,p $<0.001$} & \\
\hline Temporal outer macula with RNFL Temporal & 0.288 & 0.378 & 0.1 \\
\hline Superior outer macula with RNFL Superior & 0.306 & 0.519 & $<0.001$ \\
\hline Nasal outer macula with RNFL Nasal & 0.298 & 0.377 & 0.14 \\
\hline Inferior outer macula with RNFL Inferior & 0.402 & 0.61 & $<0.001$ \\
\hline Inner + outer macula & \multicolumn{2}{|c|}{ the correlation coefficient,p $<0.001$} & \\
\hline Temporal inner+outer macula with RNFL Temporal & 0.308 & 0.407 & 0.06 \\
\hline Superior inner + outer macula with RNFL Superior & 0.254 & 0.468 & $<0.001$ \\
\hline Nasal inner + outer macula with RNFL Nasal & 0.264 & 0.315 & 0.36 \\
\hline Inferior inner + outer macula with RNFL Inferior & 0.358 & 0.546 & $<0.001$ \\
\hline Total macular thickness/ volume & \multicolumn{2}{|c|}{ the correlation coefficient, $\mathrm{p}<0.001$} & \\
\hline Total macular thickness/ volume with RNFLTemporal & $0.316 / 0.337$ & $0.416 / 0.43$ & $0.058 / 0.0001$ \\
\hline Total macular thickness/ volume with RNFL Superior & $0.223 / 0.276$ & $0.418 / 0.485$ & $<0.001$ \\
\hline Total macular thickness/ volume with RNFL Nasal & $0.255 / 0.298$ & $0.327 / 0.403$ & $0.197 / 0.001$ \\
\hline Total macular thickness/ volume with RNFL Inferior & $0.309 / 0.367$ & $0.442 / 0.529$ & $0.01 / 0.0001$ \\
\hline Total macular thickness/ volume with RNFLThickness average & $0.38 / 0.442$ & $0.494 / 0.576$ & 0.001 \\
\hline Total macular thickness/ volume with MD (dB) & - & $0.148 / 0.187$ & - \\
\hline Total macular thickness/ volume with Cup/DiscVertical Ratio & 1.053691275 & 0.912280702 & 0.001 \\
\hline Total macular thickness/ volume withRim area $\mathrm{mm}^{2}$ & $0.167 / 0.189$ & $0.277 / 0.321$ & $0.021 / 0.001$ \\
\hline
\end{tabular}

M(SD):Mean Value and a Standard Deviation; cpRNFL: circumpapillary Retinal Nerve Fiber Layer; MD(dB): Visual Field Mean Deviation in Decibels; POAG: Primary Open Angle Glaucoma; n: number of eyes; $\mathrm{p}$ : value of correlation coefficient; $\mathrm{p}^{*}$ : value between control and POAG groups correlation coefficients

The probability of POAG risk was calculated using binary logis- $\quad$ thickness average of Inferior outer macula was $<219 \mu$. Odds ratio tic regression analysis including odds ratio and its confidence in- to have POAG increases 3 times when thickness average of Inferior terval. According to the set of obtained data, it is possible to make inner macula was $<264 \mu$, Temporal outer macula was $<212 \mu$, and prognosis that odds ratio to have POAG increases 3.5 times when Superior outer macula was $<223 \mu$ (Table 4).

Table 4: ROC test predicted values and characteristics of the distribution, according to the study groups.

\begin{tabular}{|c|c|c|c|c|c|}
\hline Variables/ Optimalvalue & $\begin{array}{c}\text { Area Under the ROC } \\
\text { Curve (\%) }\end{array}$ & Sensitivity/ Specificity (\%) & Control/ POAG (\%) & $p$ value & POAG. OR [95\% CI] \\
\hline Temporal inner & \multirow{2}{*}{63.7} & 53.9 & 33.3 & \multirow{2}{*}{$<0.001$} & \multirow{2}{*}{$2.343[1.838-2.987]$} \\
\hline macula $<250 \mu$ & & 66.7 & 53.9 & & \\
\hline Superior inner & \multirow{2}{*}{63.1} & $56.6 /$ & 35.8 & \multirow{2}{*}{$<0.001$} & \multirow{2}{*}{$2.34[1.836-2.982]$} \\
\hline macula $<262 \mu$ & & 64.2 & 56.6 & & \\
\hline Nasal inner & \multirow{2}{*}{60.6} & 65.8 & 47.3 & \multirow{2}{*}{$<0.001$} & \multirow{2}{*}{$2.142[1.673-2.744]$} \\
\hline macula $<267 \mu$ & & 52.7 & 65.8 & & \\
\hline Inferior inner & \multirow{2}{*}{64.7} & 66.8 & 42.4 & \multirow{2}{*}{$<0.001$} & \multirow{2}{*}{$2.735[2.131-3.51]$} \\
\hline macula $<264 \mu$ & & 57.6 & 66.8 & & \\
\hline Temporal outer & \multirow{2}{*}{67.4} & 63.4 & 37.8 & \multirow{2}{*}{$<0.001$} & \multirow{2}{*}{$2.856[2.231-3.655]$} \\
\hline macula $<212 \mu$ & & 62.2 & 63.4 & & \\
\hline Superior outer & \multirow{2}{*}{65.3} & 51.6 & 27.7 & \multirow{2}{*}{$<0.001$} & \multirow{2}{*}{$2.77[2.17-3.553]$} \\
\hline macula $<223 \mu$ & & 72.3 & 51.6 & & \\
\hline Nasal outer & \multirow{2}{*}{64.9} & 57.1 & 34.8 & \multirow{2}{*}{$<0,001$} & \multirow{2}{*}{$2.493[1.954-3.179]$} \\
\hline macula $<242 \mu$ & & 65.2 & 57.1 & & \\
\hline Inferior outer & \multirow{2}{*}{68.5} & 59.7 & 30.1 & \multirow{2}{*}{$<0.001$} & \multirow{2}{*}{$3.453[2.695-4.424]$} \\
\hline macula $<219 \mu$ & & 69.9 & 59.7 & & \\
\hline
\end{tabular}

POAG: Primary Open Angle Glaucoma; $\mu$ : microns; OR: Odds Ratio; CI: Confidence Interval 
The visual field mean deviation (MD dB) was classified into 3 terciles: I - MD <-3.55 dB; II - MD (-3.55 - (-2.15)) dB; III - MD $>-2.15 \mathrm{~dB}$. All provided parameters, i.e., the sectoral macular retinal thickness average in comparative analysis (the dispersion analysis (ANOVA)), I terciles (MD<-3.55 dB) were significantly lower than II and III terciles, but parameters averages of II and III treciles were significantly indifferent (Attachment(1)). ROC (Receiver Operating Characteristic) curve analysis method was used for determination Table 5: ROC test predicted values and characteristics of the distribution, according to visual field mean deviation in decibels terciles.

\begin{tabular}{|c|c|c|c|c|c|}
\hline Variables/ Optimal value & $\begin{array}{l}\text { Area Under the } \\
\text { ROC Curve: (\%) }\end{array}$ & Sensitivity/Specificity (\%) & $\begin{array}{c}\text { MD dB }>-3,55 / \\
\text { MD dB<3,55(\%) }\end{array}$ & p value & $\begin{array}{c}\text { Visual field MD } \mathrm{dB}<-3.55 \\
\text { OR[95 proc. } \mathrm{PI}]\end{array}$ \\
\hline \multicolumn{6}{|c|}{ Inner+outer macula } \\
\hline Temporal & \multirow{2}{*}{63.5} & 63.4 & 40.9 & \multirow{2}{*}{$<0.001$} & \multirow{2}{*}{$2.509[1.611-3.909]$} \\
\hline inner+outer $227 \mu$ & & 59.1 & 63.4 & & \\
\hline \multirow{2}{*}{ Superior inner + outer $238 \mu$} & \multirow{2}{*}{62.0} & 56.1 & 35.8 & \multirow{2}{*}{$<0.001$} & \multirow{2}{*}{$2.292[1.497-3.551]$} \\
\hline & & 64.2 & 56.1 & & \\
\hline \multirow{2}{*}{ Nasal inner+outer $241 \mu$} & \multirow{2}{*}{58.8} & 43.1 & 23.0 & \multirow{2}{*}{$<0.001$} & \multirow{2}{*}{$2.541[1.604-4.026]$} \\
\hline & & 77.0 & 43.1 & & \\
\hline \multirow{2}{*}{ Inferior inner+outer $237 \mu$} & \multirow{2}{*}{67.1} & 74.0 & 44.4 & \multirow{2}{*}{$<0.001$} & \multirow{2}{*}{$3.567[2.225-5.719]$} \\
\hline & & 55.6 & 74.0 & & \\
\hline \multicolumn{6}{|c|}{ Inner macula } \\
\hline \multirow{2}{*}{ Temporal inner $<230 \mu$} & \multirow{2}{*}{60.6} & 27.6 & 10.1 & \multirow{2}{*}{$<0.001$} & \multirow{2}{*}{$3.394[1.927-5.979]$} \\
\hline & & 90.9 & 27.6 & & \\
\hline \multirow{2}{*}{ Superior inner $<260 \mu$} & \multirow{2}{*}{58.4} & 63.4 & 49.4 & \multirow{2}{*}{0.01} & \multirow{2}{*}{$1.77[1.142-2.757]$} \\
\hline & & 50.6 & 63.4 & & \\
\hline \multirow{2}{*}{ Nasal inner $<252 \mu$} & \multirow{2}{*}{54.9} & 40.7 & 28.8 & \multirow{2}{*}{0.021} & \multirow{2}{*}{$1.694[1.08-2.656]$} \\
\hline & & 71.2 & 40.7 & & \\
\hline \multirow{2}{*}{ Inferior inner $<247 \mu$} & \multirow{2}{*}{62.4} & 41.8 & 21.0 & 0001 & $2662[16601251]$ \\
\hline & & 79.0 & 41.5 & $<0.001$ & $2.663[1.668-4.251]$ \\
\hline & & Outer macula & & & \\
\hline Tomorolostor & 651 & 58.5 & 34.2 & - 0001 & $2711[1742,217]$ \\
\hline lemporal outer< $204 \mu$ & 65.1 & 65.8 & 58.5 & $<0.001$ & $2.111[1.84-4.21 /]$ \\
\hline & & 72.4 & 46.7 & & \\
\hline Superior outer $<225 \mu$ & 64.5 & 53.3 & 72.4 & $<0.001$ & $2.988[1.877-4.758]$ \\
\hline Nocolouter $-222 \mu$ & 617 & 49.6 & 28.0 & - $00 \Omega 1$ & $2528[1618-2010]$ \\
\hline Nasal outer $<233 \mu$ & 01.1 & 72.0 & 49.6 & $<0.001$ & $2.528[1.618-3.949]$ \\
\hline Inferior outer $<213 \mu$ & 686 & 67.5 & 39.3 & $-0 \cap 0 \Omega 1$ & $2005 \Gamma 2020504$ \\
\hline inrerior outer < $213 \mu$ & 68.6 & 60.7 & 67.5 & $<0.001$ & $3.205[2.038-5.04]$ \\
\hline & & 42.3 & 24.1 & & \\
\hline Iotal macularthickness $<\angle 26 \mu$ & 61.5 & 73.3 & 42.3 & $<0.001$ & $2.303[1.45 /-3.641]$ \\
\hline
\end{tabular}

MD(DB): Visual Field Mean Deviation in Decibels; OR: Odds Ratio; CI: Confidence Interval; $\mu$ : microns.

The Cup/ Disc Vert Ratio were classified into 3 tercile: I - $\leq 0.425$; II - 0.426-0.576; III - $\geq 0.577$. All provided parameters, i.e., the sectoral macular retinal thickness averages in comparative analysis (the dispersion analysis (ANOVA)), III tercile (Cup/ Disc Vert Ratio $\geq 0.577$ ) were significantly lower than I and II terciles, but parameters averages of I and II treciles were significantly indifferent (Attachment (2)).

ROC (Receiver Operating Characteristic) curve analysis method was used for determination of optimal values of macular reti- of optimal values of macular retinal thickness parameters. We performed binary logistic regression analysis and assessed the odds ratio of having visual field deeper than MD - $3.55 \mathrm{~dB}$ significantly increased 3.5 time if Inferior inner+outer macular retinal thickness was less than $237 \mu$ and increased 3.4 and 3.2 times if Temporal inner macular retinal thickness was less than $<230 \mu$ and Inferior outer macular retinal thickness was less than $213 \mu$ respectively (Table 5). nal thickness parameters. We performed binary logistic regression analysis and assessed the odds ratio of having Cup/ Disc Vert Ratio bigger than 0.577 significantly increased 2.8 and 2.7 times if Inferior outer macular retinal thickness was $<219 \mu$ and Inferior inner macular, superiorinner+outer macular retinal thickness was $<255 \mu,<239 \mu$ respectively; 2.6 times if Temporal inner and Temporal inner+outer macular retinal thickness were less then $<250 \mu$ and $<251 \mu$ respectively (Table 6). 
Table 6: ROC test predicted values and characteristics of the distribution, according to Cup/ Disc Vert Ratio terciles.

\begin{tabular}{|c|c|c|c|c|c|}
\hline Variables/Optimal value & $\begin{array}{c}\text { Area Under the Curve } \\
(\%)\end{array}$ & $\begin{array}{c}\text { Sensitivity/ Specificity } \\
(\%)\end{array}$ & $\begin{array}{c}\text { Cup/ Disc Vert } \\
\text { Ratio: }<0.577 / \\
\geq 0.577(\% .)\end{array}$ & p value & $\begin{array}{l}\text { Cup/ Disc Vert Ratio } \\
\geq 0.577 \text { OR [ } 95 \text { proc. PI] }\end{array}$ \\
\hline \multicolumn{6}{|c|}{ Inner+ Outer macula } \\
\hline \multirow[t]{2}{*}{ Temporalinner+outer $<251 \mu$} & \multirow{2}{*}{65.1} & 59.1 & 34.9 & \multirow{2}{*}{$<0.001$} & \multirow{2}{*}{$2.644[2.075-3.37]$} \\
\hline & & 65.1 & 59.7 & & \\
\hline \multirow[t]{2}{*}{ Superior inner+outer $<239 \mu$} & \multirow{2}{*}{64.6} & 62.2 & 41.5 & \multirow{2}{*}{0.001} & \multirow{2}{*}{$2.759[2.179-3.492]$} \\
\hline & & 66.0 & 62.2 & & \\
\hline \multirow{2}{*}{ Nasal inner+outer $<251 \mu$} & \multirow{2}{*}{63.4} & 53.1 & 33.4 & \multirow{2}{*}{$<0.001$} & \multirow{2}{*}{$2.51[1.781-2.844]$} \\
\hline & & 66.6 & 53.1 & & \\
\hline \multirow{2}{*}{ Inferior inner+outer $<239 \mu$} & \multirow{2}{*}{66.3} & 55.6 & 30.4 & \multirow{2}{*}{$<0.001$} & \multirow{2}{*}{$2.251[1.781-2.844]$} \\
\hline & & 69.6 & 55.6 & & \\
\hline \multicolumn{6}{|c|}{ Innermacula } \\
\hline \multirow{2}{*}{ Temporal inner $<250 \mu$} & \multirow{2}{*}{64.4} & 54.9 & 31.4 & \multirow{2}{*}{$<0.001$} & \multirow{2}{*}{$2.658[2.1-3.365]$} \\
\hline & & 68.6 & 54.9 & & \\
\hline \multirow{2}{*}{ Superior inner $<265 \mu$} & \multirow{2}{*}{63.0} & 63.3 & 44.7 & \multirow{2}{*}{$<0.001$} & \multirow{2}{*}{$2.139[1.69-2.706]$} \\
\hline & & 55.3 & 63.3 & & \\
\hline \multirow[t]{2}{*}{ Nasal inner $<265 \mu$} & 61.2 & 62.6 & 44.1 & $<0.001$ & $2.125[1.681-2.687]$ \\
\hline & & 55.9 & 62.6 & & \\
\hline \multirow{2}{*}{ Inferior inner $<255 \mu$} & \multirow{2}{*}{64.4} & 46.7 & 24.5 & \multirow{2}{*}{$<0.001$} & \multirow{2}{*}{$2.701[2.12-3.442]$} \\
\hline & & 75.5 & 75.5 & & \\
\hline & & Outermacula & & & \\
\hline Temnoral outer<212u & 641 & 57.2 & 39.1 & $<0001$ & $2078[1647-26201$ \\
\hline 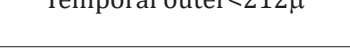 & 04.1 & 60.9 & 57.2 & 0.001 & $2.070[1.047-2.024]$ \\
\hline Sunerior outer $<225$ & 645 & 52.6 & 32.3 & $<0001$ & $2326[184-2941]$ \\
\hline superior outer $<\angle \measuredangle \zeta \mu$ & 64.5 & 67.7 & 52.6 & $<0.001$ & $2.326[1.84-2.941]$ \\
\hline Nacal outer $<248 u$ & 64.3 & 71.1 & 49.5 & $<0001$ & $2502[1958-3196]$ \\
\hline Nasal outer $<248 \mu$ & 64.3 & 50.5 & 71.1 & $<0.001$ & $2.502[1.958-3.196]$ \\
\hline Inferior outer $<219$ & 661 & 55.1 & 30.3 & $<0001$ & $283[2233-3585]$ \\
\hline 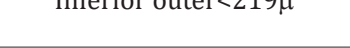 & 00.1 & 69.7 & 55.1 & $<0.001$ & $2.03[2.235-3.505]$ \\
\hline Total macular & 646 & 46.0 & 24.4 & $<0001$ & $264[2075-337]$ \\
\hline retinalthickness $<233 \mu$ & 64.6 & 75.6 & 46.3 & $<0.001$ & $2.64[2.0 / 5-3.3 /]$ \\
\hline
\end{tabular}

OR: Odds Ratio; CI: Confidence Interval; $\mu$ : Microns

\section{Discussion}

We conducted a large sample, 697 subjects (1339 eyes), case-control study [15]. The control group comprised randomly selected participants from population registry to participate in international program HAPIEE (Health, Alcohol and Psychosocial factors in Eastern Europe). The glaucomatous visual field defects of POAG group participants corresponded to stage I (early defect, MD more than or equal to $-6.00 \mathrm{~dB}$ ), based on Humphrey visual field printouts [16]. All participants underwent full ophthalmological examination and OCT of the macula, cpRNFL and OND at same visit. Our study results were based on a large sample of data. There are other studies similar to our, but they were based on limited sample size $[5-7,9,10,17-24]$.

We found significant differences in mean macular retinal thickness between control and initial POAG patients using meas- urements of retinal thickness generated with OCT. According to our data macular retinal thickness in sectors, except for the fovea, showed statistically significant thinning in the POAG group compared with the control group. Our results are in agreement with other studies comparing the macular parameters with OCT among control and POAG groups. Tan $\mathrm{O}$ et al. [9], Yamada H et al. [17] , and Dave P [24], identified statistically significant thinning of the average macular thicknesses in the POAG group compared with the control: $225.3(16.5) \mu$ and $252.6(17.7) \mu(\mathrm{p}<0.0001)$, respectively $269.6(9.8) \mu$ and $286.4(14.4) \mu(\mathrm{p}<0.001)$, respectively $275.8(16.6)$ $\mu$ and 292(9.4) $\mu(p<0.001)[9,17,24]$. Sullivan-Mee M et al. [18], also stated for both right and left eyes, RNFL and macular thickness parameters were significantly thinner in the glaucoma group versus the normal group [18].

Above mentioned investigators also found that inter eye macular thickness asymmetry had the highest diagnostic sensitivity 
(88\% at $80 \%$ specificity; $83 \%$ at $95 \%$ specificity), followed by total RNFL thickness (88\% at $80 \%$ specificity; $75 \%$ at $95 \%$ specificity) [18]. The total macular thickness average difference between the control and POAG groups were analyzed in previously discussed studies. Arvanitaki V et al. [10], as in our study examined the retinal thickness in four inner quadrants of the macula (on $1.70 \mathrm{~mm}$ radius around the macular center). Based on his study data the differences in macula retinal thickness between the control group and the POAG group were statistically significant for all quadrants and are in agreement with our results. The AROC (area under the receive operating curve) provided a summary measure of the accuracy of diagnosing glaucoma against the normal reference group and Tan 0 et al. [9], stated the macular retinal thickness average measured by FD- OCT and TD-OCT had equivalent AROC values [5].

There was a stronger correlation between relevant sectors of the cpRNFL defects and retinal thinning in the macula in the case POAG. Our results are in agreement with other studies, where were analyzed correlation between cpRNFL and the macula retinal thickness [8,21]. Greenfield DS et al. [7], concluded that the mean macular thickness was significantly correlating with cpRNFL thickness $(\mathrm{R} 2=0.38 ; \mathrm{P}<0.001)$ in the posterior segment quadrants associated with the field defect. Based on our and other authors scientific studies data $[7,8,21]$ the macular retinal thickness and cpRNFL thickness assessments were significantly associated with each other, suggesting concordance between loss of retinal ganglion cells and their axons [7]. The whole macular retinal thickness correlation with cpRNFL was analized in the previous studies; therefore we found it lacking more detailed analisys, i.e., correlation of separate sectors of macular retinal thickness with relevant sectors of cpRNFL. Arvanitaki V et al. [10], measured the macular retinal thickness and macular RNFL thickness using "Fast Macular Thickness Map" scanning protocol and "Fast RNFL Thickness (3.4)" scanning protocol on a $1.70 \mathrm{~mm}$ radius around the macular center (respectively) in all four quadrants [10]. The "Fast RNFL Thickness (3.4)" protocol was transposed on the macula from the peripapillary area. Previous studies with OCT reported significant differences between the control and POAG groups for the cp RNFL thickness $[6,7,9,18,24]$. It was interesting and surprise that the macular RNFL thickness between groups was not statistically significant for all peri-macular quadrants in Arvanitaki V et al. [10], study. But Rao HL., et al., in the similar study reported a reduction in macular RNFL thickness in glaucomatous eyes compared with normal eyes [5].

Significant differences between the two groups were found for all the parameters of macula (except for the fovea) in our study and it was in agreement with previous studies that the loss was most pronounced in the inferior region of the macula $[5,6,9,10,18,23,25]$. Accordingly, glaucoma predominantly affects the inferior periphery of the macula $[9,23,25]$. The odds ratio of having glaucoma if inferior outer macula retinal thickness average $<219 \mu$ was $3.5(95 \%$ CI, 2.038-5.04) in our study. Tan 0 et al. [9] , found the odds ratio of having glaucoma for every $10 \mu$ loss of macular retinal tissue was 2.53 (95\% CI, 1.84-3.47) using TD-OCT [5]. The AUC provides a direct measurement of discriminant power. The AUC for inferi- or outer macula retinal thickness average was 0.685 (Sensitivity/ Specificity (\%) -67.5/60.7) in our study. Tan 0 et al. [9], estimated AUC for total macular retinal thickness and it was $0.87 \pm 0.03$. Parikh RS et al. [23], as well as in our study found that the outer inferior macular retinal thickness average had the best combination of sensitivity and specificity (56\% and 79\% respectively) and outer inferior macular retinal thickness average AUC of 0.66 . Na JH et al. [25], found more significant AUC for outer inferior macular retinal thickness average -0.880 .

The total macular thickness/ volume correlation with visual mean deviation (MD dB) was very weak and the mean macular retinal thickness of the macula sectors were significantly decreased only with a corresponding visual field mean deviation worse than $-3.55 \mathrm{~dB}$. The odds ratio of having visual field MD worse than -3.55dB was 3.205 [95\% PI 2.038-5.04] and 3.394 [95\% PI 1.9275.979] if the mean of the inferior outer macular retinal thickness was $<213 \mu$ and the mean of temporal inner macular retinal thickness was $<230 \mu$ respectively. Yamada H et al. [17], Mori S et al. [22], Asrani S et al. [20], found stronger correlations of macular retinal thickness/ volume measurement and visual field MD ( $\mathrm{r}=0.475$, $\mathrm{p}<0.001),(\mathrm{r}=0.458, \mathrm{p}<0.001)$ and $(\mathrm{r}=0.54, \mathrm{p}<0.001)$ respectively. Yamada $\mathrm{H}$ et al. [17], compared normal eyes and 3 groups of glaucomatous eyes: preperimetric and perimetric glaucoma with MD better than $-6 \mathrm{~dB}$ (termed early glaucoma), and those with perimetric glaucoma with MD worse than $-6 \mathrm{~dB}$ (termed advanced glaucoma with mean of MD -15.76 (7.8) dB)). The mean of the MD was better $(-3.62 \mathrm{~dB})$ in our study and it was a reason of lower correlation between the mean of the macular retinal volume and mean of the visual field MD. Mathers K et al. [21] , also refers to macular thickness correlation with visual field deficits, with much worse MD $-6.8 \mathrm{~dB}$ score in subjects with average macular thickness $<270 \mu \mathrm{m}$.

The total thickness/ macular volume correlation with Cup/ DiscVert Ratio was weak and the mean macular retinal thickness of the macula sectors were significantly decreased only with a corresponding Cup/ Disc Vert Ratio bigger than 0.577. The retinal thickness losses correlated more strongly $(r=0.75, p<0.001)$ with severity of optic nerve disc cupping butless strongly with the severity of visual field loss $(r=0.54, p<0.001)$ in Asrani $S$ et al. [20], study. We found the odds ratio of having Cup/ Disc Vert Ratio bigger than 0.577 was 2.83 [95\% PI 2.233-3.585] and 2.701 [95\% PI 2.123.442] if the mean of the inferior outer macular retinal thickness was

$<219 \mu$ and the mean of the inferior inner macular retinal thickness was $<255 \mu$, Superior inner+outer macular retinal thickness was $<239 \mu$ respectively.

\section{Conclusion}

The thinning of the macular retinal thickness in the sectors had a significant clinical interface with changes in cpRNFL, visual field loss and OND cupping in glaucoma and may represent a surrogate indicator of retinal ganglion cell loss. Macular thickness measurements with OCT may provide a new approach for the detection and monitoring of glaucomatous damage. 


\section{Attachments}

A. The sectoral macular retinal thickness parameter distributions according to terciles of visual field mean deviation showed that sectoral macular retinal thickness averages (except Nasal inner macula) were significantly different as I tercile (<-3.55) from II and III. Sectoral macular retinal thickness averages between II and III terciles were indifferent (Table 7).

Table 7: The sectoral macular retinal thickness parameter distributions according to terciles of visual field mean deviation in decibels.

\begin{tabular}{|c|c|c|c|}
\hline \multirow{2}{*}{ Parameters microns, M(SD) } & \multicolumn{3}{|c|}{ Visual field - MD(dB), terciles } \\
\hline & I $(<-3.55),(n=131)$ & II $(-3.55-(-2.15)),(n=132)$ & III $(>-2.15),(n=132)$ \\
\hline \multicolumn{4}{|c|}{ Inner } \\
\hline \multirow{2}{*}{ Temporal innermacula } & $245.27(31.06)^{*}, * *$ & $252.26(31.06)^{*}$ & $251.31(24.8)^{* *}$ \\
\hline & \multicolumn{3}{|c|}{$\chi 2=11,982$, lls $=2, p=0,003 ; *, * * p<0.05$} \\
\hline \multirow{2}{*}{ Superior innermacula } & $258.22(32.83)^{*}, * *$ & $261.26(19,32)^{*}$ & $262.76(22.54)^{* *}$ \\
\hline & \multicolumn{3}{|c|}{$\chi 2=7.083,1 \mathrm{ls}=2, \mathrm{p}=0.029 ;{ }^{*}, * * \mathrm{p}<0.05$} \\
\hline \multirow{2}{*}{ Nasal inner macula } & $260.4(30.36)$ & $262.67(19.5)$ & $262.57(23.36)$ \\
\hline & \multicolumn{3}{|c|}{$\chi 2=2.496,11 s=2, p=0.287$} \\
\hline \multirow{2}{*}{ Inferior inner macula } & $253.63(37.41)^{*}, * *$ & $260.64(18.4)^{*}$ & $260.15(25.79)^{* *}$ \\
\hline & \multicolumn{3}{|c|}{$\chi 2=15.633,11 s=2, p<0.001 ;{ }^{*}, * * p<0.05$} \\
\hline \multicolumn{4}{|c|}{ Outer } \\
\hline \multirow{2}{*}{ Temporal outermacula } & $203.08(20.75)^{*}, * *$ & 211.91(15.95)* & $209.74(19.22)^{* *}$ \\
\hline & \multicolumn{3}{|c|}{$\chi 2=25.11$, lls $=2, p<0.001 ;{ }^{* * *} \mathrm{p}<0.05$} \\
\hline \multirow{2}{*}{ Superior outermacula } & $219.98(29.19)^{*}, * *$ & $227.12(15.7)^{*}$ & $225.31(17.32)^{* *}$ \\
\hline & \multicolumn{3}{|c|}{$\chi 2=21.474,11 s=2, p<0.001 ;{ }^{*}, * * p<0.05$} \\
\hline \multirow{2}{*}{ Nasal outermacula } & 235.13(19.7)*,** & $243.36(16.68)^{*}$ & $241.03(19.69)^{* *}$ \\
\hline & \multicolumn{3}{|c|}{$\chi 2=14.99$, lls $=2, p=0.001 ; * * * p<0.05$} \\
\hline \multirow{2}{*}{ Inferior outermacula } & $208.89(27.86)^{* * * *}$ & $220.59(16.26)^{*}$ & $217.41(21.24)^{* *}$ \\
\hline & \multicolumn{3}{|c|}{$\chi 2=37.265,11 s=2, p<0.001 ; *, * * p<0.05$} \\
\hline \multicolumn{4}{|c|}{ Inner+ Outer } \\
\hline \multirow{2}{*}{ Temporal inner +outer macula } & $448.35(50.13)^{* * *}$ & $464.18(32.7)^{*}$ & $461.05(42.98)^{* *}$ \\
\hline & \multicolumn{3}{|c|}{$\chi 2=19.738,11 \mathrm{~s}=2, \mathrm{p}<0.001 ;{ }^{*}, * * \mathrm{p}<0.05$} \\
\hline \multirow{2}{*}{ Superior inner +outer macula } & $478.2(59.88)^{*}, * *$ & $488.39(32.66)^{*}$ & $488.07(37.98)^{* *}$ \\
\hline & \multicolumn{3}{|c|}{$\chi 2=14.321, \mathrm{lls}=2, \mathrm{p}=0.001 ;{ }^{*}, * * \mathrm{p}<0.05$} \\
\hline \multirow{2}{*}{ Nasal inner + outermacula } & $495.53(46.24)^{* * * *}$ & $506.03(33.64)^{*}$ & $503.6(40.95)^{* *}$ \\
\hline & \multicolumn{3}{|c|}{$\chi 2=8.343$, lls $=2, p=0.015 ; * * * p<0.05$} \\
\hline \multirow{2}{*}{ Inferior inner + outer macula } & $462.52(63.27)^{* * *}$ & $481.22(32.78)^{*}$ & $477.55(45.57)^{* *}$ \\
\hline & \multicolumn{3}{|c|}{$\chi 2=30.441, \mathrm{lls}=2, \mathrm{p}<0.001 ;{ }^{*}, * * \mathrm{p}<0.05$} \\
\hline
\end{tabular}

M(SD): Mean Value and a Standard Deviation; $\mathrm{MD}(\mathrm{dB})$ : Visual Field Mean Deviation in Decibels; n: number of eyes; p: value on the basis of nonparametrical Kruskal-Wallis (X2) and Mann- Whitney test, lls: number of degrees of freedom *, **,***.

B. Parameter distributions according to terciles of Cup/ DiscVert Ratio terciles showed that sectoral macular retinal thickfrom I and II terciles (I and II were significantly indifferent) (Table 8).

ness averages of III tercile $(\geq 0.577)$ were significantly different

Table 8: Parameter distributions according to terciles of Cup/DiscVert Ratio terciles.

\begin{tabular}{|c|c|c|c|}
\hline \multirow{2}{*}{ Parameters microns, M(SD) } & \multicolumn{3}{|c|}{ Parameter Cup/ Disc VertRatioterciles } \\
\hline & I $(\leq 0.425),(n=450)$ & II $(0.426-0.576),(n=455)$ & III ( $\geq 0.577),(n=449)$ \\
\hline \multicolumn{4}{|c|}{ Inner } \\
\hline \multirow{2}{*}{ Temporal inner macula } & $259.25(26.28)^{*}$ & $257.17(19.17)^{* *}$ & $249.56(25.5)^{* * * *}$ \\
\hline & \multicolumn{3}{|c|}{$\chi 2=73.954$, lls $=2, p<0.001 ; *^{*}, * * p<0.001$} \\
\hline \multirow{2}{*}{ Superior inner macula } & $270.19(27.42)^{*}$ & $267.99(19.99)^{* *}$ & $260.26(21.55)^{* * *}$ \\
\hline & \multicolumn{3}{|c|}{$\chi 2=61.333,1 \mathrm{ll}=2, \mathrm{p}<0.001 ;{ }^{*}, * * \mathrm{p}<0.001$} \\
\hline
\end{tabular}




\begin{tabular}{|c|c|c|c|}
\hline \multirow{2}{*}{ Nasal inner macula } & $269.77(24.78)$ & $267.21(22.49)$ & $260.54(24.52)^{*}, * *$ \\
\hline & \multicolumn{3}{|c|}{$\chi 2=46.257$, lls $=2, \mathrm{p}<0.001 ;{ }^{*}, * * \mathrm{p}<0.001$} \\
\hline \multirow{2}{*}{ Inferior inner macula } & $268.72(23.45)^{*}$ & $267.34(20.47)^{* *}$ & $258.16(28.0)^{* * *}$ \\
\hline & \multicolumn{3}{|c|}{$\chi 2=73.356,1 \mathrm{l} s=2, \mathrm{p}<0.001 ; * * * * \mathrm{p}<0.001$} \\
\hline \multicolumn{4}{|c|}{ Outer } \\
\hline \multirow{2}{*}{ Temporal outer macula } & $218.27(20.76)^{*}$ & $216.88(15.88)^{* *}$ & $209.86(19.71)^{* * *}$ \\
\hline & \multicolumn{3}{|c|}{$\chi 2=70.81,1 \mathrm{ls}=2, \mathrm{p}<0.001 ;{ }^{*}, * * \mathrm{p}<0.001$} \\
\hline \multirow{2}{*}{ Superior outer macula } & $234.2(23.35)^{*}$ & $232.61(15.92)^{* *}$ & $224.38(18.48)^{*}, * *$ \\
\hline & \multicolumn{3}{|c|}{$\chi 2=73.74,11 s=2, p<0.001 ;{ }^{*}, * * p<0.001$} \\
\hline \multirow{2}{*}{ Nasal outer macula } & $249.74(18.15)^{*}$ & $249.72(19.74)^{* *}$ & $239.96(20.05)^{*}, * *$ \\
\hline & \multicolumn{3}{|c|}{$\chi 2=72.033$, lls $=2, \mathrm{p}=0.001 ;{ }^{*}, * * \mathrm{p}<0.001$} \\
\hline \multirow{2}{*}{ Inferior outer macula } & $227.28(18.93)^{*}$ & $227.44(22.51)^{* *}$ & $217.02(21.84)^{* * *}$ \\
\hline & \multicolumn{3}{|c|}{$\chi 2=90.668,1 \mathrm{ll}=2, \mathrm{p}<0.001 ;{ }^{*}, * * \mathrm{p}<0.001$} \\
\hline \multicolumn{4}{|c|}{ Inner + Outer } \\
\hline \multirow{2}{*}{ Temporal inner+outermacula } & $477.53(44.85)^{*}, * *$ & $474.06(33.15)^{* *}$ & $459.42(43.06)^{* * *}$ \\
\hline & \multicolumn{3}{|c|}{$\chi 2=81.749$, lls $=2, \mathrm{p}<0.001 ; * * * \mathrm{p}<0.001$} \\
\hline \multirow{2}{*}{ Superior inner+outer macula } & $504.39(48.96)^{*}$ & $500.6(34.01)^{* *}$ & $484.64(37.55)^{* * *}$ \\
\hline & \multicolumn{3}{|c|}{$\chi 2=75.378$, lls $=2, p<0.001 ;{ }^{*}, * * p<0.001$} \\
\hline \multirow{2}{*}{ Nasal inner+outer macula } & $519.5(39.92)^{*}$ & $516.94(39.9)^{* *}$ & $500.5(41.27)^{* * *}$ \\
\hline & \multicolumn{3}{|c|}{$\chi 2=63.779,1 \mathrm{l}=2, \mathrm{p}<0.001 ;{ }^{*}, * * \mathrm{p}<0.001$} \\
\hline \multirow{2}{*}{ Inferior inner+outer macula } & $495.98(39.62)^{*}$ & $494.78(38.55)^{* *}$ & $475.18(47.69)^{*}, * *$ \\
\hline & \multicolumn{3}{|c|}{$\chi 2=94.201$, lls $=2, p<0.001 ; *^{*}, * * 0.001$} \\
\hline
\end{tabular}

M(SD): Mean Value and a Standard Deviation; $n$ : Number of eyes; $p$ : value on the basis ofnonparametricalKruskal-Wallis $(\chi 2)$ and Mann-Whitney test, lls: number of degree of freedom*, **, ***.

\section{References}

1. Sung KR, Wollstein G, Kim NR, Na JH, Nevins JE, et al. (2012) Macular assessment using optical coherence tomography for glaucoma diagnosis. Br J Ophthalmol 96(12): 1452-1455.

2. Hoffmann EM, Zangwill LM, Crowston JG, Weinreb RN (2007) Optic disk size and glaucoma. Surv Ophthalmol 52(1): 32-49.

3. Jonas JB (2011) Role of cerebrospinal fluid pressure in the pathogenesis of glaucoma. Acta Ophthalmol 89(6): 505-514.

4. Neubauer AS, Krieglstein TR, Chryssafis Ch, Thiel M, Kampik A (2006) Comparison of optical coherence tomography and fundus photography for measuring the optic disc size. Ophthalmic Physiol Opt 26(1): 13-18.

5. Tan O, Chopra V, Lu ATH, Schuman JS, Ishikawa H, et al. (2009) Detection of macular ganglion cell loss in glaucoma by Fourier-domain optical coherence tomography. Ophthalmology 116(12): 2305-2314.

6. Rao HL, Babu JG, Addepall UK, Senthiand S, Garuda CS (2012) Retinal nerve fiber layer and macular inner retina measurements by spectral domain optical coherence tomography in Indian eyes with early glaucoma. Eye 26(1): 133-139.

7. Greenfield DS, Bagga H, Knighton RW (2003) Macular thickness changes in glaucomatous optic neuropathy detected using optical coherence tomography. Arch Ophthalmol 121(1): 41-46.

8. Gupta D, Asrani S (2016) Macular thickness analysis for glaucoma diagnosis and management. Taiwan Journal of Ophthalmology 6(1): 3-7.

9. Tan O, Li G, Lu ATH, Varma R, Huang D (2008) Mapping of macular substructures with optical coherence tomography for glaucoma diagnosis. Ophthalmology 115(6): 949-956.

10. Arvanitaki V, Tsilimbaris MK, Pallikaris A, Moschandreas I, Minos E, et al. (2012) Macular retinal and nerve fiber layer thickness in early glaucoma: Clinical correlations. Middle East African Journal of Ophthalmology 19(2): 204-210.

11. Buteikiene D, Paunksnis A, Barzdziukas V, Zaliuniene D, Balciuniene JV, et al. (2012) Correlations between digital planimetry and optical coherence tomography, confocal scanning laser ophthalmoscopy in assessment of optic disc parameters. Medicina 48(3): 150-158.

12. Oduntan OA, Mashige KP, Raliavhegwa Makhado M (2009) A comparison of two methods of log MAR visual acuity data scoring for statistical analysis. S Afr Optom 68(3): 155-163.

13. Bencic G, Zoric-Geber M, Saric D, Corak M, Mandic Z (2005) Clinical importance of the lens opacities classification system III (LOCS III) in phacoemulsification. Coll Antropol 1: 91-94.

14. Stratus OCT User Manual (2007) Carl Zeiss Meditec.

15. Bochmann F, Johnson Z, Azuara-Blanco A (2007) Sample size in studies on diagnostic accuracy in ophthalmology: A literature survey. $\mathrm{Br}$ J Ophthalmol 91(7): 898-900.

16. Mills RP, Budenz DL, Lee PP, Noecker JR, Walt JG, et al. (2006) Categorizing the stage of glaucoma from pre-diagnosis to end-stage disease. Am J Ophthalmol 141(1): 24-30.

17. Yamada H, Hangai M, Nakano N, Takayama K, Kimura Y, et al. (2014) Asymmetry analysisof macular inner retinal layers for glaucoma diagnosis. American Journal of Ophthalmology 158(6): 1318-1329.

18. Sullivan-Mee M, Ruegg CC, Pensyl D, Halverson K, Qualls C (2013) Diagnostic precision of retinal nerve fiber layer and macular thickness asymmetry parameters for identifying early primary open-angle glaucoma. American Journal of Ophthalmology 156(3): 567-577.

19. Inuzuka H, Kawase K, Sawada A, Aoyama Y, Yamamoto T (2013) Macular retinal thickness in glaucoma with superior or inferior visual hemifield defects. J Glaucoma 22(1): 60- 64. 
20. Asrani S, Challa P, Herndon L, Lee P, Stinnett S, et al. (2003) Correlation among retinal thickness, optic disc, and visual field in glaucoma patients and suspects: A pilot study. J Glaucoma 12(2): 119-128.

21. Mathers K, Rosdahl JA, Asrani S (2014) Correlation of macular thickness with visual fields in glaucoma patients and suspects. J Glaucoma 23(2): 98-104.

22. Mori S, Hangai M, Sakamoto A, Yoshimura N (2010) Spectral-domain optical coherence tomography measurement of macular volume for diagnosing glaucoma. J Glaucoma 19(8): 528-534.

\section{(C) (P) This work is licensed under Creative}

Submission Link: https://biomedres.us/submit-manuscript.php
23. Parikh RS, Parikh SR, Thomas R (2010) Diagnostic capability of macular parameters of Stratus OCT 3 in detection of early glaucoma. $\mathrm{Br}$ J Ophthalmol 94(2): 197-201.

24. Dave P, Shah J (2015) Diagnostic accuracy of posterior pole asymmetry analysis parameters of spectralis optical coherence tomography in detecting early unilateral glaucoma. Indian J Ophthalmol 63(11): 837842.

25. Na JH, Sung KR, Baek S, Sun JH, Lee Y (2011) Macular and retinal nerve fiber layer thickness: Which is more helpful in the diagnosis of glaucoma? Invest Ophthalmol Vis Sci 52(11): 8094-8101.

$\begin{array}{ll}\text { BIOMEDICAL } & \text { Assets of Publishing with us } \\ \text { RESEARCHES } & \text { - Global archiving of articles } \\ \text { - Immediate, unrestricted online access } & \text { Rigorous Peer Review Process } \\ & \text { - Authors Retain Copyrights } \\ & \end{array}$

